\title{
Diagnosis of Influenza: Only a Problem of Coding?
}

\author{
Emanuele Amodio Fabio Tramuto Claudio Costantino Vincenzo Restivo \\ Carmelo Maida Giuseppe Calamusa Francesco Vitale
}

Department of Sciences for Health Promotion and Mother-Child Care 'G. D'Alessandro', University of Palermo, Palermo, Italy

\section{Key Words}

Influenza · Hospital discharge records · Influenza-like illness · Epidemiology

\section{Abstract}

Objective: To evaluate the characteristics of hospital discharge diagnoses of influenza measured by using specific International Classification of Diseases, 9th Revision, Clinical Modification (ICD-9 CM) codes. Subjects and Methods: The study was conducted for the 3 years 2007, 2008 and 2011. The database included (1) administrative and clinical data on Sicilian patients admitted to acute care hospitals and (2) data from the influenza virological surveillance of 10 European countries (FluNet database). All Sicilian patients diagnosed with at least 1 ICD-9 CM code for influenza (487.0, 487.1 and 487.9) were considered influenza cases. Results: Overall, 2,880 patients with an ICD-9 CM code attributable to influenza were hospitalized in Sicily: 2,119 (73.6\%) were admitted from November to April, whereas 761 (26.4\%) were admitted from May to October. In the 3 years studied, the analyzed European influenza surveillance systems recorded a peak of laboratory-confirmed influenza activity from November to

\begin{tabular}{ll}
\hline KARGER & $\begin{array}{l}\text { ( } 2014 \text { S. Karger AG, Basel } \\
1011-7571 / 14 / 0236-0568 \$ 39.50 / 0\end{array}$ \\
$\begin{array}{l}\text { E-Mail karger@karger.com } \\
\text { www.karger.com/mpp }\end{array}$ & $\begin{array}{l}\text { This is an Open Access article licensed under the terms of the } \\
\text { Creative Commons Attribution-NonCommercial 3.0 Un- } \\
\text { ported license (CC BY-NC) (www.karger.com/OA-license), } \\
\text { applicable to the online version of the article only. Distribu- } \\
\text { tion permitted for non-commercial purposes only. }\end{array}$
\end{tabular}

April with 36,753 (99.7\%) influenza cases, whereas only 124 cases $(0.3 \%)$ were observed from May to October. Conclusions: In Sicily, more than one quarter of all hospital admissions with an ICD-9 CM code for influenza were observed in the months with a negligible circulation of influenza viruses. Our findings show that several hospital discharge records included ICD-9 CM codes for influenza with low levels of sensitivity, specificity and/or appropriateness for clinical information and support the need for improving medical education on the epidemiology and hospital management of influenza cases.

(c) 2014 S. Karger AG, Basel

\section{Introduction}

Seasonal influenza is one of the most important infectious diseases in the world, causing up to 500,000 deaths per year [1]. However, influenza-associated mortality represents only the 'tip' of the iceberg, since a consistently larger number of persons infected with influenza virus exhibit self-limited, uncomplicated, acute febrile respiratory symptoms. Globally, both 'mild' and 'severe' influ- 
enza cases account for several billions of days of illness each year, hundreds of millions of days of work lost and millions of hospitalizations that have to be added to the list of costs attributable to influenza [2,3].

Despite this consistently huge year-to-year burden, the epidemiology and prevention of influenza remain topics of discussion [4], even if healthcare personnel are not always fully aware of their importance [5]. Some healthcare workers' attitudes to and perceptions of influenza vaccination seem to confirm the presence of several misconceptions and a general lack of knowledge/education on these issues [6].

Contrary to international evidence-based public health guidelines and recommendations, very low vaccination coverage rates against influenza have recently been reported among healthcare workers throughout the world [7-9], including Italy [9-11]. Equally, refusals of immunization are often due to misconceptions as well as the belief that the vaccine can actually cause influenza, one's feeling of being healthy and never being at risk of influenza infection or skepticism about the potential effectiveness of vaccination [12-15]. Apparently, the lack of welldefined clinical characteristics of influenza infection and the overlap with influenza-like illnesses (ILIs) [16] could contribute to missed diagnoses and ultimately lead to questioning the value of specific vaccinations.

In order to establish preventive measures against influenza, public health workers make wide use of many surveillance systems of the International Classification of Diseases (ICD) to detect and monitor unusual disease activity by extracting nonspecific clinical data from information systems in clinical settings [17].

Therefore, using a large population-based database, in this study, we sought to assess the relationship between the ICD codes for influenza used in hospital discharge records collected in Sicily and influenza circulation as observed by several European virological surveillance systems.

\section{Subjects and Methods}

The study was conducted for the 3 years 2007, 2008 and 2011; 2009 and 2010 were excluded from the analysis because, in these years, an exceptionally active circulation of pandemic influenza A (H1N1) virus was observed during the warm months in Italy [18] and around the world [19]. The following data were assessed: (1) administrative and clinical data on Sicilian patients admitted to acute care hospitals and (2) data from the influenza virological surveillance systems of 10 European countries (FluNet database). Neither ethics approval nor individual written consent by adults and pediatric patients was requested according to the Italian law on anonymous aggregate data.

Medical Diagnosis of Influenza in Hospital Settings

\section{Hospital Discharge Records}

All the data included in the analyses were obtained from the Health Agency of the Sicilian government. The Regional Hospital Discharge Database, which was established in 1994, consists of complete data on hospitalizations from both public and private hospitals. Each hospital discharge record includes demographic information (gender and date of birth), admission and discharge dates, discharge status (categorized as 'discharged/transferred' or 'expired') and up to 6 discharge diagnoses (1 principal diagnosis and 5 secondary diagnoses) coded according to the ICD, 9th Revision, Clinical Modification (ICD-9 CM) that was used in Sicily in the years under study. All patients diagnosed with at least 1 ICD-9 CM code of those reported below, either as the principal or as a secondary diagnosis, were considered displaying symptoms suggestive of influenza (ICD-9 CM code 487): (1) 487.0 influenza with pneumonia, (2) 487.1 influenza with other respiratory manifestations and (3) 487.8 influenza with other manifestations. The ICD9 CM codes for specified viral pneumonia (480.0-480.3 and 480.8) and unspecified viral pneumonia (480.9) were also included in the analyses in order to assess the presence of coding patterns.

Based on the comorbidity classification by Elixhauser et al. [20], the most frequent comorbidities were retrieved using the following ICD-9 CM codes as the principal or a secondary diagnosis: diabetes mellitus (250.00-250.93), congestive heart failure (398.91, 402.11, 402.91, 404.11, 404.13, 404.91, 404.93 and 428), chronic obstructive pulmonary disease (490-492.8, 493.00-493.91, 494505 and 506.4), renal failure (403.11, 403.91, 404.12, 404.92, 585, 586, V42.0, V45.1, V56.0 and V56.8), liver disease (070.32, 070.33, $070.54,456.0-456.2,571.0,571.2-571.9,572.3,572.8$ and V42.7) and cancer (196-199, 140-172, 174, 175, 179-195 and V10).

Hospitalizations of Sicilian residents that occurred outside the region were also included in the analysis, whereas multiple hospitalizations due to transfers were combined. Admissions for transferred patients were followed until discharge or death.

\section{Influenza Virological Surveillance}

Virological data from influenza surveillance were obtained by consulting the World Health Organization FluNet database, which monitors influenza activity through the National Influenza Centers of the Global Influenza Surveillance Network (GISN) and from national influenza reference laboratories that collaborate with the GISN. Included in this study for analysis were the numbers of influenza cases detected in 10 European countries arbitrarily chosen on the basis of the completeness of their surveillance for each year and their geographic proximity to Sicily (Italy, Croatia, France, Germany, Greece, Poland, Portugal, Romania, Spain and Switzerland) [21]. The percentages of specimens per 4 weeks that tested positive for influenza A and B viruses in 2007, 2008 and 2011 were used for comparing the virologically confirmed influenza circulation and monthly influenza-associated hospitalizations.

\section{Statistical Analysis}

All data were analyzed using the $\mathrm{R}$ statistical software package. The significance level was set at $\mathrm{p}<0.05$ (two-tailed). Data on influenza activity are presented as relative frequencies (percentages per 4 weeks), whereas influenza cases in Sicilian hospitalized patients are summarized as absolute frequencies (cases per month). Categorical variables were analyzed using the $\chi^{2}$ test (MantelHaenszel) or the Fisher exact test as indicated. 
Table 1. Characteristics of patients hospitalized in Sicily with an ICD-9 CM diagnosis of influenza

\begin{tabular}{|c|c|c|c|}
\hline & $\begin{array}{l}\text { Influenza season }{ }^{\mathrm{a}} \\
(\mathrm{n}=2,119 ; 73.6 \%)\end{array}$ & $\begin{array}{l}\text { Not influenza season } \\
(\mathrm{n}=761 ; 26.4 \%)\end{array}$ & $\begin{array}{l}\text { Total } \\
(\mathrm{n}=2,880 ; 100 \%)\end{array}$ \\
\hline \multicolumn{4}{|l|}{$\operatorname{Age}^{* * *}$} \\
\hline $0-14$ years & $1,064(50.2)$ & $231(30.3)$ & $1,295(45.0)$ \\
\hline $15-24$ years & $199(9.4)$ & $92(12.1)$ & $291(10.1)$ \\
\hline $25-64$ years & $556(26.2)$ & $260(34.2)$ & $816(28.3)$ \\
\hline$\geq 65$ years & $300(14.2)$ & $178(23.4)$ & $478(16.6)$ \\
\hline \multicolumn{4}{|l|}{ Sex } \\
\hline Male & $1,123(53.0)$ & $389(51.1)$ & $1,512(52.5)$ \\
\hline Female & $996(47.0)$ & $372(48.9)$ & $1,368(47.5)$ \\
\hline \multicolumn{4}{|l|}{ Hospital stay* } \\
\hline $0-2$ days & $679(32.0)$ & $203(26.7)$ & $882(30.6)$ \\
\hline $3-5$ days & $954(45.0)$ & $354(46.5)$ & $1,308(45.4)$ \\
\hline$\geq 6$ days & $486(23.0)$ & $204(26.8)$ & $690(24.0)$ \\
\hline \multicolumn{4}{|l|}{ Presence of comorbidities } \\
\hline Diabetes mellitus** & $92(4.3)$ & $52(6.8)$ & $144(5.0)$ \\
\hline Congestive heart failure & $13(0.6)$ & $6(0.8)$ & $18(0.7)$ \\
\hline Chronic obstructive pulmonary disease & $94(4.4)$ & $24(3.1)$ & $118(4.1)$ \\
\hline Renal failure & $24(1.1)$ & $5(0.7)$ & $29(1.0)$ \\
\hline Liver disease & $60(2.8)$ & $31(4.1)$ & $91(3.2)$ \\
\hline Cancer & $25(1.2)$ & $13(1.7)$ & $38(1.3)$ \\
\hline \multicolumn{4}{|l|}{ Discharge status* } \\
\hline Discharged/transferred & $2,106(99.4)$ & $761(100)$ & $2,867(99.5)$ \\
\hline Expired & $13(0.6)$ & $0(0)$ & $13(0.5)$ \\
\hline
\end{tabular}

Values denote numbers with percentages in parentheses. ${ }^{*} \mathrm{p}<0.05 ;{ }^{* *} \mathrm{p}<0.01 ;{ }^{* * *} \mathrm{p}<0.001$. ${ }^{\mathrm{a}}$ From November to April. ${ }^{b}$ From May to October.

\section{Results}

During 2007, 2008 and 2011, a total of 3,181,822 hospital discharge records were collected among Sicilian subjects: $1,203,554(37.83 \%)$ in $2007,1,093,690(34.37 \%)$ in 2008 and $884,578(27.80 \%)$ in 2011 . A total of 2,880 patients were hospitalized with an ICD-9 CM code attributable to influenza: 1,092 (37.92\%) in 2007, 973 (33.78\%) in 2008 and $815(28.3 \%)$ in 2011. Of these 2,880 patients, 1,295 (45.0\%) were children aged $0-14$ years and had a hospital stay from 3 to 5 days (45.4\%; table 1$)$. Influenza was equally distributed between males and females $(\mathrm{M} / \mathrm{F}$ ratio $=1.1$ ), and $0.5 \%$ of all the patients with influenza died during their hospital stay. Of the 2,880 patients, 2,119 (73.6\%) were admitted from November to April (781 in 2007, 733 in 2008 and 605 in 2011), whereas 761 (26.4\%) were admitted from May to October (311 in 2007, 240 in 2008 and 210 in 2011).

Patients hospitalized from November to April were significantly younger $(26.4 \pm 26.7$ vs. $36.1 \pm 28.2$ years; $\mathrm{p}=0.001$ ), had a shorter hospital stay (median: 3 vs. 4 days; $\mathrm{p}=0.01$ ), had a lower prevalence of diabetes (4.3 vs. $6.8 \% ; \mathrm{p}<0.01)$ and were at significantly higher risk of death $(0.6$ vs. $0 \% ; \mathrm{p}<0.03)$ than those admitted from May to October.

The annual number of hospitalizations observed in Sicily for influenza and for specified $(\mathrm{n}=112 ; 12.1 \%)$ and unspecified $(\mathrm{n}=811 ; 87.9 \%)$ viral pneumonia are shown in figure 1. Both the hospitalizations for specified and those for unspecified pneumonia did not show a marked seasonality; however, the hospitalizations for influenza peaked in January and February.

The number of hospitalizations with an ICD-9 CM code for influenza infection and the percentages of laboratory-confirmed influenza cases observed during the study years in the 10 European countries are summarized in figure 2 . In the 3 years studied, the analyzed European influenza surveillance systems recorded a peak of laboratory-confirmed influenza activity from November to April with 36,753 (99.7\%) influenza cases, whereas only 124 cases $(0.3 \%)$ were observed from May to October. 


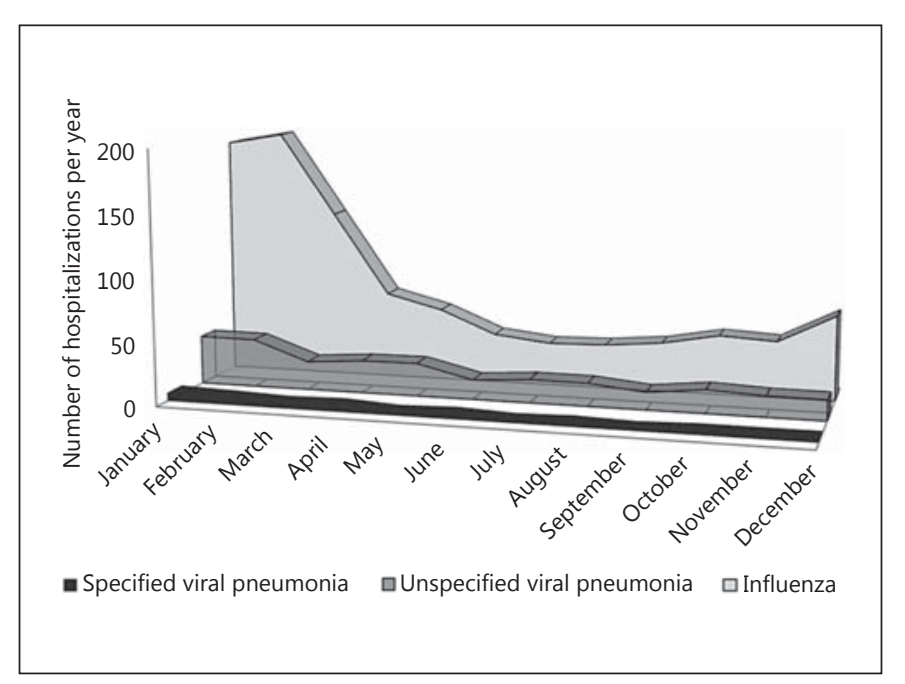

Fig. 1. Annual number of patients hospitalized in Sicily with ICD$9 \mathrm{CM}$ codes for influenza infection as well as for specified and unspecified viral pneumonia.

\section{Discussion}

The present study showed that in Sicily, in the 3 years studied, a mean of $960 \pm 139$ patients were hospitalized for influenza, accounting for an incidence rate of more than 20 cases per 100,000 residents per year. Moreover, our results suggest three main considerations that seem to support the hypothesis that ICD-9 CM codes for influenza were often included in hospital discharge records with low levels of sensitivity, specificity and/or appropriateness for clinical information.

Firstly, the hospitalization rates for influenza in Sicily were far lower than those reported previously (more than 100 influenza-associated hospitalizations per 100,000 person-years) [22]. The difference could partly be attributed to some common limitations of all passive surveillance systems, such as underdiagnosis or deficiencies in reporting, that were different in other geographic settings and, ultimately, affected the quality of the ICD-9 CM coding in terms of completeness and accuracy in our study. Also, other problems that could not be excluded were related to the hospital discharge records, which show a simple, timely and inexpensive picture of the epidemiology of diseases as they provide a complete record of all hospitalizations and standardized diagnosis code lists that enable a secular trend analysis of incident disease for a given population.

Secondly, more than $25 \%$ of all influenza-associated hospitalizations in our study occurred from May to Oc-
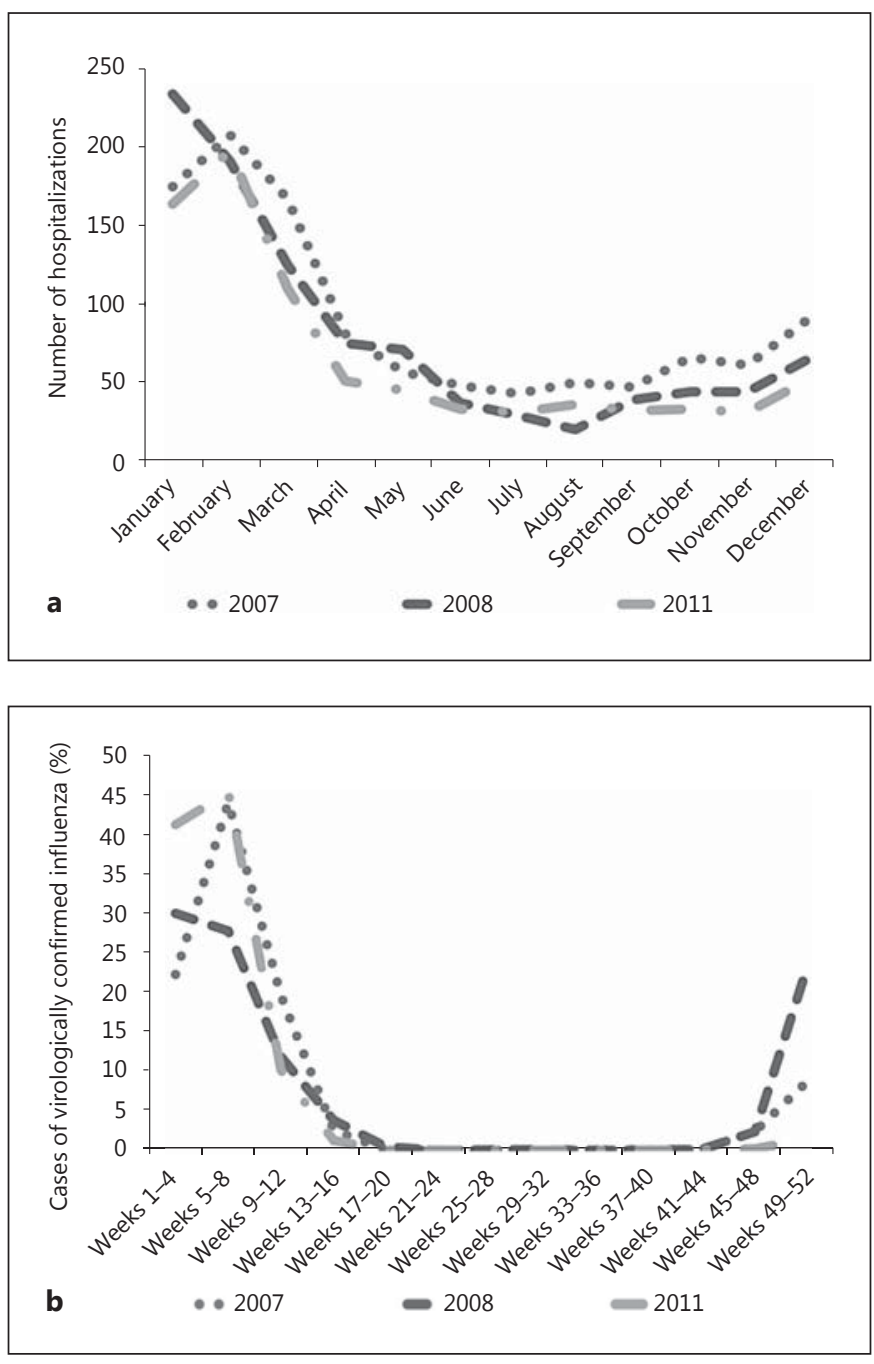

Fig. 2. a Number of patients hospitalized in Sicily (2007, 2008 and 2011) with an ICD-9 CM code for influenza infection. b Aggregate percentage of laboratory-confirmed influenza cases in Italy, Croatia, France, Germany, Greece, Poland, Portugal, Romania, Spain and Switzerland.

tober, thus in periods during which, both in Europe and in Italy, there is no active circulation of influenza viruses $[23,24]$. Moreover, in our database, the low number of specified and unspecified viral pneumonia codes found in the records during the influenza season could suggest a misclassification of the different viral diseases, supporting the low specificity of influenza codes observed by other authors who reported values ranging from $43 \%$ for the ICD-9 CM code 487.8 to $75 \%$ for the ICD-9 CM code 487.0 [25].

Thirdly, our study patients diagnosed with influenza and hospitalized outside the influenza seasons were sig- 
nificantly older and had longer hospital stays with respect to influenza-associated hospitalizations during the remaining part of the year, whereas they did not differ in the prevalence of the investigated co-morbidities (with the exception of diabetes). These latter findings could be linked to the possibly increased severity of the disease as well as an etiologic agent other than an influenza virus. Further investigations may be required for answering these questions.

The three previous considerations support the hypothesis of common medical habits that, in the absence of laboratory confirmation, do not consider epidemiologic information on influenza in their differential diagnoses of influenza and other respiratory infections. Although this attitude may not significantly influence patient care, it is of paramount importance for medical education, since it supports the false conviction that influenza vaccination protects against ILIs, not knowing that ILIs can be caused by several respiratory microorganisms (e.g. rhinovirus, adenovirus, respiratory syncytial virus, parainfluenza virus and human metapneumovirus) other than influenza type A and B viruses. For the same reasons, a misclassification between influenza and ILI could also generate the belief that a respiratory syndrome subsequent to influenza vaccination, which, in Italy, is prevented by inactivated vaccines, may be due to immunization. Finally, an inaccurate diagnosis of respiratory viral pathogens may impair patient outcomes and increase healthcare costs while promoting antibiotic stewardship.

Unfortunately, this study suffers from two possible main limitations. A first limitation is the lack of an etio- logic laboratory confirmation of influenza cases and the absence of active virological influenza surveillance in Italy during the warmer period of the year. As a second point, it should be considered that the use of a local regional dataset could reduce the generalizability of our data to different contexts.

\section{Conclusion}

The findings of this study show that, in each of the years investigated, influenza was an important cause of hospitalizations in Sicily, especially during winter. Also, the analysis of the hospital discharge records shows that ICD-9 CM codes for influenza were probably used with low levels of sensitivity, specificity and/or appropriateness for clinical information and supports the need for improving medical education on these topics.

\section{Acknowledgements}

The authors are grateful to Dr. Sergio Buffa and Dr. Giovanni Allegra of the Information Center of the Sicilian government for their contribution to the realization of this study.

\section{Disclosure Statement}

The authors have no conflicts of interest to declare.

\section{References}

1 Thompson WW, Shay DK, Weintraub E, et al: Mortality associated with influenza and respiratory syncitial virus in the United States. JAMA 2003;289:179-186.

$\checkmark 2$ Nichol KL, Treanor JJ: Vaccines for seasonal and pandemic influenza. J Infect Dis 2006; 194(suppl 2):S111-S118.

-3 Behbehani N, Mahmood A, Mokaddas EM, et al: Significance of atypical pathogens among community-acquired pneumonia adult patients admitted to hospital in Kuwait. Med Princ Pract 2005; 14:235-240.

-4 Jefferson T, di Pietrantonj C, Rivetti A, et al: Vaccines for preventing influenza in healthy adults. Cochrane Database Syst Rev 2010; 7:CD001269.

5 Thomas RE, Jefferson T, Lasserson TJ: Influenza vaccination for healthcare workers who work with the elderly: systematic review. Vaccine 2010;29:344-356.
6 Hollmeyer H, Hayden F, Mounts A, et al: Review: interventions to increase influenza vaccination among healthcare workers in hospitals. Influenza Other Respir Viruses 2013;7: 604-621.

7 Mereckiene J, Cotter S, D'Ancona F, et al: Differences in national influenza vaccination policies across the European Union, Norway and Iceland 2008-2009. Euro Surveill 2010; 15:pii: 19700.

8 Bellia C, Setbon M, Zylberman P, et al: Healthcare worker compliance with seasonal and pandemic influenza vaccination. Influenza Other Respir Viruses 2013;7:97-104.

-9 Quach S, Pereira JA, Kwong JC, et al: Immunizing health care workers against influenza: a glimpse into the challenges with voluntary programs and considerations for mandatory policies. Am J Infect Control 2013;41:10171023.
10 Amodio E, Anastasi G, di Pasquale M, et al: Influenza vaccination among healthcare workers and absenteeism from work due to influenza-like illness in a teaching hospital in Palermo. Ital J Public Health 2010;7:311-318.

11 Amodio E, Anastasi G, Marsala MG, et al: Vaccination against the 2009 pandemic influenza A (H1N1) among healthcare workers in the major teaching hospital of Sicily (Italy). Vaccine 2011;29:1408-1412.

12 Hofmann F, Ferracin C, Marsh G, et al: Influenza vaccination of healthcare workers: a literature review of attitudes and beliefs. Infection 2006;34:142-147.

13 Amodio E, Tramuto F, Maringhini G, et al: Are medical residents a 'core group' for future improvement of influenza vaccination coverage in health-care workers? A study among medical residents at the University Hospital of Palermo (Sicily). Vaccine 2011;29:8113-8117. 
14 Bonfiglioli R, Vignoli M, Guglielmi D, et al: Getting vaccinated or not getting vaccinated? Different reasons for getting vaccinated against seasonal or pandemic influenza. BMC Public Health 2013;13:1221.

15 Bish A, Yardley L, Nicoll A, et al: Factors associated with uptake of vaccination against pandemic influenza: a systematic review. Vaccine 2011;29:6472-6484.

16 Thomas RE: Is influenza-like illness a useful concept and an appropriate test of influenza vaccine effectiveness? Vaccine 2014;32:21432149.

17 Cadieux G, Buckeridge DL, Jacques A, et al: Accuracy of syndrome definitions based on diagnoses in physician claims. BMC Public Health 2011;11:17.
18 Tramuto F, Maida CM, Bonura F, et al: Surveillance of hospitalised patients with influenza-like illness during pandemic influenza $\mathrm{A}$ (H1N1) season in Sicily, April 2009-December 2010. Euro Surveill 2011;16:pii: 19957.

19 Owayed AF, Husain EH, Al-Khabaz A, et al: Epidemiology and clinical presentation of pandemic influenza A (H1N1) among hospitalized children in Kuwait. Med Princ Pract 2012;21:254-258.

20 Elixhauser A, Steiner C, Harris DR, et al: Comorbidity measures for use with administrative data. Med Care 1998;36:8-27.

21 Global Influenza Surveillance and Response System (GISRS) - World Health Organization. Accessed in 2013 at http://www.who.int/ influenza/gisrs_laboratory/updates/summaryreport/en/index.html.
22 Thompson WW, Shay DK, Weintraub E, et al: Influenza-associated hospitalizations in the United States. JAMA 2004;292:1333-1340.

23 Tramuto F, Maida CM, Magliozzo F, et al: Occurrence of a case of influenza A (H1N1) pdm09 and B co-infection during the epidemic season 2012-2013. Infect Genet Evol 2014; 23:C95-C98.

24 Campa A, Quattrocchi M, Guido M, et al: Ten-year (1999-2009) epidemiological and virological surveillance of influenza in South Italy (Apulia). Influenza Res Treat 2010;2010: 642492.

25 Marsden-Haug N, Foster VB, Gould PL, et al: Code-based syndromic surveillance for influenzalike illness by International Classification of Diseases, Ninth Revision. Emerg Infect Dis 2007;13:207-216. 